\title{
INFLUÊNCIA DE FATORES DE RISCO NA MORTALIDADE POR DOENÇAS INFECCIOSAS E PARASITÁRIAS
}

Márcio Pioli

Doutorando no Programa de Pós-graduação Stricto sensu em Promoção de Saúde da Universidade de Franca (UNIFRAN), Franca (SP), Brasil.

\section{Regina Helena Pires}

Doutora; Docente do Programa de Pós-graduação Stricto sensu em Promoção de Saúde da Universidade de Franca (UNIFRAN), Franca (SP), Brasil.

E-mail: regina.pires@unifran.edu.br

\section{Salvador Boccaletti Ramos}

Doutor; Docente no Programa de Pós-graduação Stricto sensu em Promoção de Saúde da Universidade de Franca (UNIFRAN), Franca (SP), Brasil.

\section{Carlos Henrique Martins}

Doutor; Docente no Programa de Pós-graduação Stricto sensu em Promoção de Saúde da Universidade de Franca (UNIFRAN), Franca (SP), Brasil.

\section{Lucas Eduardo de Oliveira Aparecido}

Doutorando na Universidade Estadual Paulista Júlio de Mesquita Filho (UNESP), Faculdade de Ciências Agrárias e Veterinárias, Brasil.

\section{José Eduardo Zaia}

Doutor; Docente na Universidade do Estado de Minas Gerais (UEMG), Brasil.
RESUMO: O Brasil tem grande extensão e características socioeconômicas, demográficas e culturais distintas. Sendo assim, em algumas regióes, as doenças infecciosas e parasitárias (DIPs) ainda fazem parte do cotidiano da população. Desse modo, objetivou-se avaliar se as DIPs, nos municípios que constituem a Superintendência Regional de Saúde (SRS) de Alfenas (MG), têm relação com fatores de risco selecionados. Trata-se de um estudo transversal de base populacional, de caráter descritivo e abordagem quantitativa, utilizando-se dados secundários obtidos no censo demográfico (2010) e empregando-se a análise de correlação de Pearson. Todos os indicadores de saúde e educação estudados, correlacionaram-se significativamente com a mortalidade por DIPs. O sistema de abastecimento de água por rede geral de distribuição, juntamente com o número de esgotamento sanitário por rede geral de esgoto ou pluvial, foram as variáveis dos indicadores de saneamento básico que demonstraram os mais elevados índices de correlação. Dentre os indicadores relativos à economia, o rendimento nominal mensal até $1 / 4$ de salário mínimo ou sem rendimento mensal influenciaram significativamente a mortalidade por DIPs e, entre os indicadores de mortalidade infantil, somente o número de nascidos com peso inferior a $2500 \mathrm{~g}$ demonstrou relação com as DIPs. Os resultados mostraram que as condições ambientais e de saneamento assim como as condições socioeconômicas dos residentes nos domićlios servidos por esses bens, aliadas à divulgação de medidas básicas de higiene pessoal, doméstica e comunitária impactam na saúde da população, refletindo na morbidade e mortalidade por enfermidades de diferentes etiologias, incluindo-se às DIPs. Dessa maneira, o conhecimento da prevalência e das principais variáveis associadas pode fornecer subsídio para dimensão do problema, planejamento e implementação de ações de promoção à saúde e à prevenção de agravos do processo saúdedoença.

PALAVRAS-CHAVE: Doenças Transmissíveis; Saúde Pública; Fatores de Risco; Mortalidade.

\section{INFLUENCE OF MORTALITY RISK FACTORS BY INFECTIOUS AND PARASITE DISEASES}

ABSTRACT: Due to Brazil's huge extension and to different social, economic, demographic and cultural conditions, several regions in Brazil feature infectious and parasite diseases (IPDs). Current analysis assesses whether IPDs in the municipalities of the Health Regional Superintendence of Alfenas MG Brazil are related to specific risk factors. Current populated-based transversal, descriptive and quantitative study employs secondary data from the 2010 demographic census, by Pearson's co-relationship. All health and educational indicators significantly co-relate themselves with mortality due to IPDs, The 
water supply system and the number of sewerage and rainwater per network unit were the variables of basic sanitation indexes with the highest co-relation scores. In the case of economic indicators, the monthly income of half the minimum wage or without any monthly earnings significantly affected IPD-based mortality. Only the number of births weighing less than $2500 \mathrm{~g}$ had any relationship to IPDs. Results showed environmental and sanitation conditions, coupled to the social and economic factors of residents in homes with such characteristics, plus the dissemination of basic measures for personal, home and community hygiene impact population's health. The latter influences morbidity and mortality by diseases of different etiologies, including IPDs. Knowledge on the prevalence and main associated variables may be a help in evaluating the problem and the planning and implementation of activities for the health and prevention of health-disease relationships.

KEY WORDS: Communicable Diseases; Public Health; Risk Factors; Mortality.

\section{INTRODUÇÃO}

Por um longo tempo as doenças infecciosas e parasitárias (DIPs) prevaleceram nas populações (SZWARCWALD et al., 2004), embora, nos últimos anos, tenha havido um decréscimo de mortes decorrentes de DIPs (POSSAS; 2001). Entretanto, devido ao Brasil ser um país heterogêneo, de grande extensão territorial e, ainda, apresentar características socioeconômicas, demográficas e culturais distintas (CAMPELO; GONCALVES; DONADI, 2005), as DIPs ainda fazem parte do cotidiano das famílias brasileiras, sendo um dos grandes problemas de saúde pública (PIUVEZAM et al., 2015).

As DIPs causam um impacto social ao país, uma vez que estão diretamente associadas ao índice de pobreza, às condições de falta de infraestrutura urbana, habitação, alimentação e higiene precárias (SCHRAMM et al., 2004). Além disso, a degradação do meio ambiente pelo homem, principalmente nas áreas mais pobres, onde a urbanização vem ocorrendo de maneira muito rápida e, pode-se dizer, na maioria das vezes de forma não planejada, não controlada e, principalmente subfinanciada, fez com que fosse excedida a capacidade financeira e administrativa das cidades em prover infraestrutura e serviços essenciais como água, saneamento, coleta e destinação adequada de lixo, serviços de saúde, além de empregos e moradia, e em assegurar segurança e controle do meio ambiente (PIGNATTI, 2004), o que tem refletido na qualidade de vida da população. Além disso, o desmatamento e a ocupação de regiões ribeirinhas expandiram as áreas de transmissão de doenças tais como a febre amarela; doenças controladas como a dengue ressurgiram em proporções epidêmicas; doenças restritas à zona rural, tais como a leishmaniose e a hanseníase, propagaramse para a zona urbana e, doenças como a tuberculose, mostraram quadro de persistência na população ao longo dos anos.

Índices demonstram que os países que alcançaram o desenvolvimento industrial e melhoraram as condições de vida da população, mostraram alterações no padrão epidemiológico (GIGANTE; MOURA; SARDINHA, 2009) e, consequentemente, redução das DIPs. Szwarcwald et al. (2004), relatam que para um país reduzir o índice de doenças, inicialmente faz-se necessária a redução da mortalidade geral, de modo a aumentar a expectativa de vida da população. Redução do analfabetismo e da mortalidade infantil também são importantes, uma vez que as questões socioeconômicas da população são fatores relevantes que influenciam os índices de risco para aquisição de doenças. Melhorias sanitárias e ambientais, implantação de novas tecnologias, ampliação do acesso aos serviços de saúde e medidas de controle de doenças, incluindo-se as vacinas e antibióticos, também contribuíram para a modificação do padrão de ocorrência das DIPs, embora as mesmas tenham prevalecido principalmente na população menos privilegiada (ISHITANI et al., 2006; GIGANTE; MOURA; SARDINHA, 2009).

$\mathrm{Na}$ literatura são encontrados alguns trabalhos avaliando as DIPs e fatores de risco tais como o de Cavalcante et al. (2016), que ao avaliarem o foco de tais doenças nos hospitais do Distrito Federal, relataram que quase metade das internações que ocorreram por condições sensíveis à atenção primária foram por DIPs (45,14\%), realçando a influência deste grupo de doenças no perfil da morbimortalidade da população brasileira. Felli et al. (2015), ao estudarem a exposição dos trabalhadores de enfermagem aos diversos tipos de doenças observaram que a grande consequência do afastamento de funcionários está relacionada aos problemas respiratórios e às DIPs. 
Assim, objetivou-se com este trabalho avaliar se as DIPs têm relação com fatores de risco selecionados, sendo o estudo realizado com dados secundários referentes aos municípios que constituem a Superintendência Regional de Saúde (SRS) de Alfenas (MG).

\section{METODOLOGIA}

O estudo foi realizado com os dados da Superintendência Regional de Saúde (SRS) de Alfenas
(MG), a qual é constituída pelos municípios de Alfenas, Alterosa, Arceburgo, Areado, Bandeira do Sul, Botelhos, Cabo Verde, Campestre, Campo do Meio, Campos Gerais, Carmo do Rio Claro, Carvalhópolis, Conceição da Aparecida, Divisa Nova, Fama, Guaranésia, Guaxupé, Juruaia, Machado, Monte Belo, Muzambinho, Nova Resende, Paraguaçu, Poço Fundo, São Pedro da União e Serrania, sendo sua localização geográfica mostrada na Figura 1.
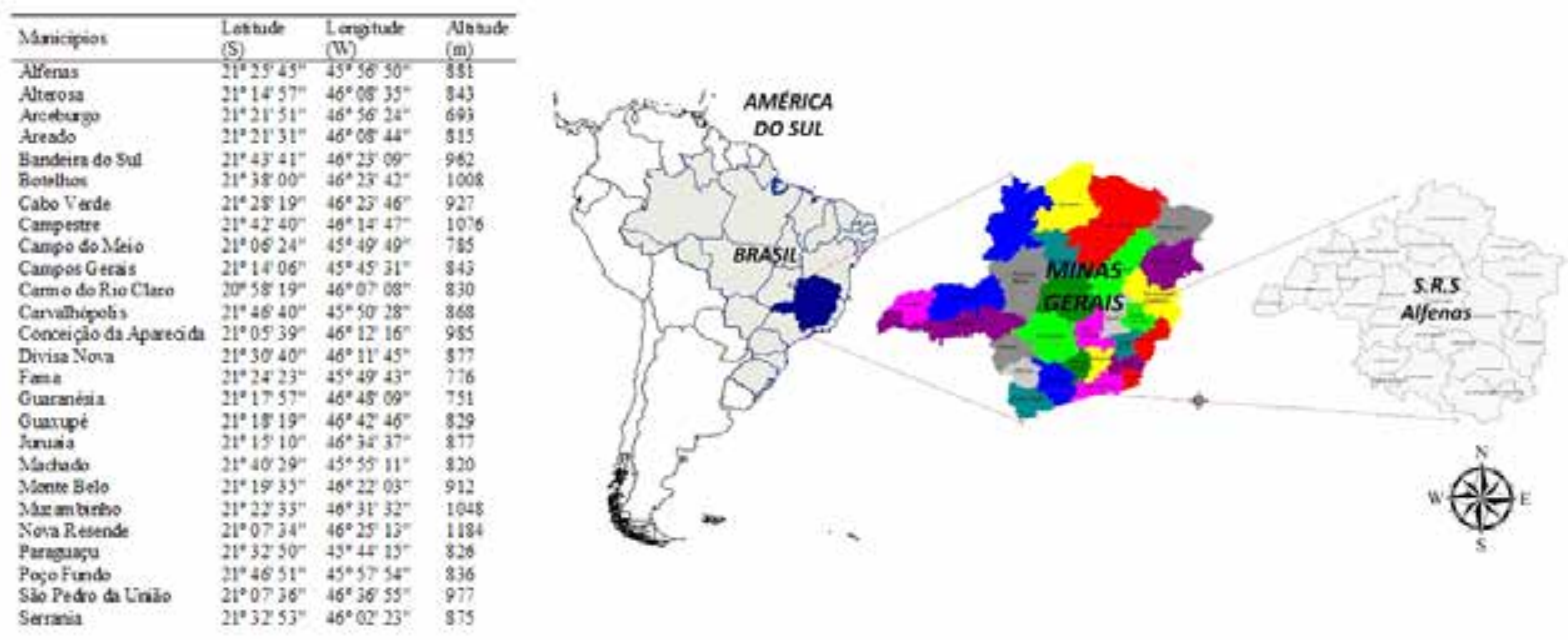

Figura 1. Localização geográfica da Superintendência Regional de Saúde (SRS) de Alfenas, Estado de Minas Gerais, segundo dados do IBGE (2010).

Trata-se de um estudo transversal de base populacional, de caráter descritivo e abordagem quantitativa, utilizando-se como método de pesquisa o levantamento de dados secundários, obtidos por meio do Instituto Brasileiro de Geografia e Estatística (IBGE Censo demográfico), tendo-se como base o ano de 2010. Os dados utilizados foram os casos de mortalidade por DIPs, juntamente com os fatores de risco de todos os municípios, subdivididos nas seguintes classes:

1) Indicadores de saneamento: sistema de abastecimento de água por rede geral de distribuição; sistema de abastecimento de água por poço ou nascente na propriedade; abastecimento de água por poço ou nascente fora da propriedade; abastecimento de água por rio, açude ou igarapé; abastecimento por água da chuva armazenada em cisternas; outras formas de abastecimento de água e número de esgotamento sanitário por rede geral de esgoto ou pluvial.

2) Indicadores de saúde: número de estabelecimentos de saúde que prestam serviço ao Sistema Único de Saúde (SUS); número de estabelecimento de saúde SUS e número de leitos para internações.

3) Indicadores educacionais: Pessoas com 10 anos ou mais que não frequentam a escola; pessoas com 10 anos ou mais sem instrução e fundamental incompleto; pessoas de 25 anos ou mais de idades residentes em domicílios particulares sem instrução e fundamental incompleto e a taxa de analfabetismo. 
4) Indicadores de renda: Pessoas de 10 anos ou mais de idade com classes de rendimento nominal mensal até 1/4 de salário mínimo; pessoas de 10 anos ou mais de idade com classes de rendimento nominal mensal sem rendimento total; porcentagem de extremamente pobres; renda per capita e rendimento médio dos ocupados com 18 anos ou mais.

5) Indicadores de saúde infantil: Porcentagem de nascidos com peso inferior a 2500.g; porcentagem de mortalidade infantil e porcentagem de esperança de vida ao nascer.

A hipótese de normalidade foi analisada pelo teste de Kolmogorov-Smirnov $(p \leq 0,05)$, seguindo-se à análise de correlação. Para avaliar se havia correlação entre mortalidade por DIPs e os fatores de risco foi estimada a correlação de Pearson (r), as quais foram consideradas significativas quando o valor de $p<0,05$. As análises foram geradas por meio do software Microsoft Excel ${ }^{\circledR}$, em rotina de Visual Basic for Applications (VBA).

\section{RESULTADOS}

A análise da correlação dos fatores de risco com a mortalidade pelas DIPs na Superintendência Regional de Saúde (SRS) de Alfenas, mostrou que os fatores de risco apresentaram relações direta e indiretamente proporcionais, além de níveis de correlações diversos.

Os níveis mais elevados de correlação (r) foram observados nos fatores de riscos associados ao saneamento básico (sistema de abastecimento de água por rede geral de distribuição e esgotamento sanitário); à saúde (todos); à educação (pessoas com 10 anos ou mais que não frequentavam a escola, pessoas com 10 anos ou mais sem instrução e fundamental incompleto, pessoas de 25 anos ou mais de idade residentes em domicílios particulares sem instrução e fundamental incompleto) e à renda (pessoas de 10 anos ou mais de idade com classe de rendimento nominal mensal até $1 / 4$ de salário mínimo e pessoas de 10 anos ou mais de idade com classe de rendimento nominal mensal sem rendimento total), os quais apresentaram um r acima de 0,82 (Quadro 1).

Quadro 1. Estimativas de correlação linear de Pearson entre número de mortes por doenças infecto-parasitárias e grupos de indicadores estudados

(continua)

\begin{tabular}{|c|c|c|}
\hline $\begin{array}{l}\text { Grupos de fatores de } \\
\text { risco }\end{array}$ & Indicador & Estimativa da correlação \\
\hline \multirow{7}{*}{ Saneamento básico } & Sistema de abastecimento de água por rede geral de distribuição & $0,82 *$ \\
\hline & Sistema de abastecimento de água por poço ou nascente na propriedade & $0,56 *$ \\
\hline & Abastecimento de água por poço ou nascente for a da propriedade & 0,06 \\
\hline & Abastecimento de água por rio, açude ou igarapé & $0,60^{*}$ \\
\hline & Abastecimento por água da chuva armazenada em cisternas & $-0,06$ \\
\hline & Outras formas de abastecimento de água & $0,63^{*}$ \\
\hline & Esgotamento sanitário por rede geral de esgoto ou pluvial & $0,82 *$ \\
\hline \multirow{3}{*}{ Saúde } & Número de estabelecimentos de saúde que prestam serviço ao SUS & $0,85^{*}$ \\
\hline & Número de Estabelecimento de saúde SUS & $0,84^{*}$ \\
\hline & Número de leitos para internações & $0,83^{*}$ \\
\hline \multirow{4}{*}{ Educação } & Pessoas com 10 anos ou mais que não frequentam a escola & $0,84 *$ \\
\hline & Pessoas com 10 anos ou mais sem instrução e fundamental incompleto & $0,86^{*}$ \\
\hline & $\begin{array}{l}\text { Pessoas de } 25 \text { anos ou mais de idade residentes em domicílios } \\
\text { particulares sem instrução e fundamental incompleto }\end{array}$ & $0,84^{*}$ \\
\hline & Taxa de analfabetismo & $0,39 *$ \\
\hline
\end{tabular}




\begin{tabular}{|l|l|c|}
\hline $\begin{array}{l}\text { Grupos de fatores de } \\
\text { risco }\end{array}$ & \multicolumn{1}{|c|}{ Indicador } & Estimativa da correlação \\
\hline \multirow{5}{*}{ Renda } & $\begin{array}{l}\text { Pessoas de } 10 \text { anos ou mais de idade classe de rendimento nominal } \\
\text { mensal até 1/4 de salário mínimo }\end{array}$ & $0,85^{*}$ \\
\cline { 2 - 4 } & $\begin{array}{l}\text { Pessoas de } 10 \text { anos ou mais de idade com classe de rendimento nominal } \\
\text { mensal sem rendimento total }\end{array}$ & $0,84^{*}$ \\
\cline { 2 - 4 } & Porcentagem de extremamente pobres & $-0,11$ \\
\cline { 2 - 4 } & Renda per capita & 0,28 \\
\cline { 2 - 4 } & Rendimento médio dos ocupados 18 anos ou mais & 0,35 \\
\hline \multirow{5}{*}{ Mortalidade infantil } & Porcentagem de nascidos com peso inferior a 2500g & $0,72^{*}$ \\
\cline { 2 - 4 } & Porcentagem de mortalidade infantil & $-0,19$ \\
\cline { 2 - 4 } & Porcentagem de esperança de vida ao nascer & 0,18 \\
\hline
\end{tabular}

Nota: $*(p<0,05)$

Fonte: (IBGE, 2010)

Para selecionar quais variáveis mais influenciaram a mortalidade por DIPs, foram ordenadas as correlações dos fatores de risco em módulo e em ordem decrescente. Conforme mostrado no Quadro 1, o fator de risco educacional, representado pelo número de pessoas com fundamental incompleto foi a variável que mais influenciou o nível de mortalidade ( $r=0,86)$, enquanto que a variável com menor nível de correlação foi a percentual de mortalidade infantil $(r=-0,19)$, fator relacionado ao poder econômico da população.

\section{DISCUSSÃO}

O reconhecimento da importância do saneamento e de sua associação com a saúde humana já é conhecida desde à antiguidade, sendo ressaltada por vários autores. Martins et al. (2000; 2002) ao avaliarem o impacto do saneamento básico na saúde da população do Vale do Ribeira e do Médio Paranapanema (SP), constataram que houve queda da mortalidade por doenças infecciosas intestinais e helmintíases a partir de 1983, ano em que começou no interior do Estado de São Paulo o Programa de Saneamento do Interior (SANIN). Tal implementação possibilitou o aumento da extensão da rede coletora de esgotos na periferia da cidade de Itapetininga, contribuindo de maneira decisiva para a redução das DIPs.
Além disso, Carmo et al. (2003) relataram que o efeito da melhoria das condições de saneamento se traduz não somente na diminuição de doenças infecciosas que oneram o sistema de saúde, mas também na prevenção para outras. Entretanto, os autores também salientam que os benefícios não são alcançados de forma homogênea, por todos os grupos populacionais, o que contribui para a manutenção de carga persistente de morbidade.

Por sua vez, Szwarcwald et al. (2004) observaram que a ampliação da cobertura populacional por redes de abastecimento de água e por sistemas de esgotamento sanitário melhoravam o índice de mortalidade por doenças infecciosas. Atualmente, o saneamento básico tem recebido maior atenção governamental com uma quantidade significativa de recursos sendo investidos. No entanto, ainda não estão definidas, de maneira clara, as atribuições de cada esfera governamental, o que tem prejudicado tanto o planejamento como a eficácia dos recursos aplicados (LEONETI; PRADO; OLIVEIRA, 2011).

Embora a implantação de sistemas de abastecimento de água e de esgotamento sanitário proporcione benefícios gerais para a saúde da população, de maneira direta e indireta, esses são resultantes, primordialmente, da cobertura de atendimento médico à localidade atendida. Desta maneira, foram avaliados também indicadores de saúde (Quadro 1) nos municípios estudados, notando-se que todas as variáveis se correlacionavam significativamente $(p<0,05)$ com as DIPs. 
O número de estabelecimentos de saúde que prestam serviço ao SUS, o número de estabelecimento de saúde SUS e o número de leitos para internações demonstraram níveis de correlação de 0,85; 0,84 e 0,83, respectivamente. Esses resultados somam-se aos de Paes (2004), que estudou o perfil da mortalidade por DIP na população de mais de 65 anos no Brasil e sua associação com variáveis socioeconômicas no período de 1980 a 1995. Foi destacado que o número de leitos hospitalares apresentava elevada correlação com as DIPs, embora não tenha sido constatada alteração significativa no percentual deóbitos por DIP na população idosabrasileira, no período estudado, para ambos os sexos. No entanto, foi verificada uma importante transição no período, caracterizada pela diminuição tanto da tuberculose pulmonar como da tripanossomíase e aumento da septicemia para ambos os sexos. Além disso, altas taxas de internações por condições sensíveis à atenção primária têm sido associadas às deficiências na cobertura dos serviços e/ou à baixa resolutividade da atenção primária (BERMUDEZ; BAKER, 2005; ANSARI LADITKA; LADITKA, 2006). No Brasil, a criação do Sistema Único de Saúde (SUS) em 1988 e, em 1994, com a institucionalização do Programa Saúde da Família (PSF), atual Estratégia Saúde da Família (ESF), tem remodelado todo o sistema assistencial no país, buscando-se uma maior racionalidade na utilização dos recursos, propiciando um melhor acesso à atenção primária em todo o país.

Também, a maioria dos indicadores da educação (Quadro 1) mostraram-se significativos com o índice de mortalidade por DIPs. Essa relação também foi destacada por Nestil e Goldbaum (2007) que analisaram a vulnerabilidade para doenças infecciosas em crianças cuidadas em ambientes coletivos (creches públicas e privadas). Os autores relataram que os funcionários de creches, em sua maioria, integram uma população vulnerável de mulheres jovens em idade reprodutiva, frequentemente de baixo nível educacional e socioeconômico, sendo que poucas receberam treinamento nos diferentes aspectos da assistência à criança relacionado à saúde, incluindo as práticas de manuseio de alimentos. A educação de usuários e prestadores de cuidado pode ser a melhor estratégia para diminuir a transmissão de doença nestes ambientes
(NESTII; GOLDBAUM, 2007).

Nas avaliações, observou-se que o número de pessoas com 10 anos ou mais que não frequentam a escola; o número de pessoas com 10 anos ou mais sem instrução e fundamental incompleto e o número de pessoas de 25 anos ou mais de idade residentes em domicílios particulares sem instrução e fundamental incompleto foram os que evidenciaram os maiores níveis de correlação no grupo de fatores de risco relacionados à educação. Martins et al. (2002), destacam que pessoas com maior nível de escolaridade, apresentam consequentemente maior nível de higiene, o que contribui para reduzir os índices de mortalidade por DIPs. Ainda, a baixa escolaridade materna, repercutindo na impossibilidade de discernir sinais de gravidade dos sintomas, aliada a dificuldade de acesso aos serviços de saúde e à carência de redes de apoio às famílias tendem a aumentar a mortalidade por DIP nessa população.

A desigualdade social é uma das causas que interferem nos índices de doenças brasileiras (FELLI et al., 2015), sendo um fator determinante na elaboração das taxas de morbidade da população (POSSAS, 2001). Dentre os indicadores de renda, observou-se que as variáveis, números de pessoas de 10 anos ou mais de idade com classe de rendimento nominal mensal até $1 / 4$ de salário mínimo e o número de pessoas de 10 anos ou mais de idade com classe de rendimento nominal mensal sem rendimento total foram as variáveis estatisticamente significativas $\quad(p<0,05), \quad$ evidenciando elevadas correlações, sendo 0,85 e 0,84, respectivamente (Quadro 1). Esses resultados contribuíram com os resultados de Paes (2004) que destacaram que o aumento do número de pessoas com baixo rendimento nominal mensal tem correlação significativa com o índice de mortalidade por DIPs. Além disso, Martins et al. (2002) relataram que a população de baixa renda geralmente sofre com as doenças infecciosas devido às condições de precariedade em que vivem, fragilizando-a e a tornando susceptível à um maior número de doenças.

As correlações dos indicadores de mortalidade infantil (Quadro 1) com os índices de DIPs demonstraram que somente a variável, nascidos com peso inferior a $2500 \mathrm{~g}$, foi significativa $(p<0,05)$. Essa correlação evidencia que quanto maior o número de crianças nascidas abaixo do 
peso de $2500 \mathrm{~g}$, maior necessariamente é o número de mortes por DIPs. Nossos resultados somam-se aos de Teixeira e Guilhermino (2006) que também observaram que o número de crianças abaixo do peso foi o indicador de mortalidade infantil com maior significância. Ainda, Carneiro et al. (2012) destacam que crianças nascidas abaixo do peso ideal precisam de maiores cuidados para sobreviverem e, Martins et al. (2002), destacam a forte tendência de aumento do nível de mortalidade por DIPs quando as crianças nascem abaixo do peso ideal.

\section{CONCLUSÃO}

De maneira geral, os fatores de risco decorrente da inter-relação entre urbanização, desemprego, exclusão social, pobreza e da relação humana com o meio ambiente encontrados na região de Alfenas (MG), são semelhantes aos da população brasileira em geral, onde se observa uma interconexão entre padrões de morbi-mortalidade contemporâneos e doenças infecto-parasitárias. O reconhecimento da dinâmica característica de cada território bem como a identificação das especificidades e vulnerabilidade de cada região, por parte da gestão pública, pode fornecer subsídio para dimensão do problema, planejamento e implementação de ações de promoção à saúde e à prevenção de agravos do processo saúde-doença.

\section{AGRADECIMENTOS}

Agradecemos à Coordenação de Aperfeiçoamento de Pessoal de Nível Superior (Capes) pela concessão de taxa à Márcio Pioli (Processo n. ${ }^{\circ}$ 1271185).

\section{REFERÊNCIAS}

ANSARI, Z.; LADITKA, J. N.; LADITKA, S. B. Access to health care and hospitalization for ambulatory care sensitive conditions. Med Care Res Rev., v. 63, p. 719741, 2006.

BERMUDEZ, D.; BAKER, L. The relationship between
SCHIP enrollment and hospitalizations for ambulatory care sensitive conditions in California. $\mathbf{J}$ Health Care Poor Underserved, v. 16, p. 96-110, 2005.

CAMPELO, V.; GONCALVES, M. A. G.; DONADI, E. A. Mortalidade por doenças infecciosas e parasitárias no Município de Teresina-PI (Brasil), 1971-2000. Rev Bras Epidemiol., v. 8, n.1, p.31-40, 2005.

CARMO, E. H.; BARRETO, M. L.; SILVA, J. B. Mudanças nos padrões de morbimortalidade da população brasileira: os desafios para um novo século. Epidemiol Serv Saúde, v. 12, n. 2, p.63-75, 2003.

CARNEIRO, J. A.; VIEIRA, M. M.; REIS, T. C.; CALDEIRA, A. P. Fatores de risco para a mortalidade de recém-nascidos de muito baixo peso em Unidade de Terapia Intensiva Neonatal. Rev Paul Pediatr., v. 30, n. 3, p.369-376, 2012.

CAVALCANTE, D. M.; OLIVEIRA, M. R. F.; REHEM, T. C. M. S. Internações por condições sensíveis à atenção primária: estudo de validação do SIH/SUS em hospital do Distrito Federal, Brasil, 2012. Cad Saúde Pública, v. 32, n. 3, e00169914, 2016.

FELLI, V. E. A.; COSTA, T. F.; BAPTISTA, P. C. P.; GUIMARÃES, A. L. O.; ANGINONI, B. M. Exposição dos trabalhadores de enfermagem às cargas de trabalho e suas consequências. Rev Esc Enferm USP, v. 49, n. 2, p.98$105,2015$.

GIGANTE, D. P.; MOURA, E. C.; SARDINHA, L. M. V. Prevalência de excesso de peso e obesidade e fatores associados, Brasil, 2006. Rev Saúde Pública. v. 43, n. 2 , p.83-89, 2009.

IBGE. Instituto Brasileiro de Geografia e Estatística. Censo demográfico 2010: indicadores. Disponível em: <http:// www.ibge.gov.br/home/mapa_site.php\#indicadores $>$. Acesso em: 18 mar. 2016.

ISHITANI, L. H.; FRANCO, G. C.; PERPETUO, I. H. O.; FRANÇA, E. Desigualdade social e mortalidade precoce por doenças cardiovasculares no Brasil. Rev Saúde Pública, v. 40, n. 4, p.684-691, 2006.

LEONETI, A. B.; PRADO, E. L.; OLIVEIRA, S. V. W. B. Saneamento básico no Brasil: considerações sobre investimentos e sustentabilidade para o século XXI. Rev 
Adm Pública, v. 45, n. 2, p.331-348, 2011.

MARTINS, G.; BORANGA, J. A.; FRANÇA, J. T. L.; PEREIRA, H. A. S. L. Impacto de sistemas de abastecimento de água na saúde pública. In: CONGRESSO INTERAMERICANO DE ENGENHARIA SANITÁRIA E AMBIENTAL, 27., 2000, Porto Alegre. Anais... Porto Alegre: [s.n.], 2000.

MARTINS, G.; BORANGA, J. A.; LATORRE, M. R. D. O.; PEREIRA, H. A. L. S. Impacto do saneamento básico na saúde da população de Itapetininga - SP, de 1980 a 1997. Eng Sanit Ambient., v. 4, p.161-188, 2002.

NESTI, M. M. M.; GOLDBAUM, M. As creches e préescolas e as doenças transmissíveis. J Pediatr., v. 83, n. 4, p.299-312, 2007.

PAES, N. A. A mortalidade por doenças infecciosas e parasitárias na população idosa brasileira. Rev Panam Salud Publica, v. 15, n. 4, p.233-241, 2004.

PIGNATTI, M. G. Saúde e ambiente: as doenças emergentes no Brasil. Ambient Soc., v. 7, n. 1, p.133$147,2004$.

PIUVEZAM, G.; FREITAS, M. R.; COSTA, J. V.; FREITAS, P. A.; CARDOSO, P. M. O. Fatores associados ao custo das internações hospitalares por doenças infecciosas em idosos em hospital de referência na cidade do Natal, Rio Grande do Norte. Cad Saúde Colet., v. 23, n. 1, p.63-68, 2015.

POSSAS, C. Social ecosystem health: confronting the complexity and emergence of infectious diseases. Cad de Saúde Pública, v. 17, n. 1, p. 31-41, 2001.

SCHRAMM, J.M.A.; OLIVEIRA, A. F.; LEITE, I. C.; VALENTE, J. G.; GADELHA, A. M. J.; PORTELA, M. C. et al. Transição epidemiológica e o estudo da carga de doenças no Brasil. Ciênc. Saúde Coletiva, v. 9, n. 4, p.897-908, 2004.

SZWARCWALD, C.L.; VIACAVA, F.; VASCONCELLOS, M. T. L.; LEAL, M. C.; AZEVEDO, L. O.; QUEIROZ, R. S. B. de et al. Pesquisa mundial de Saúde 2003: o Brasil em números. Radis, v. 23, p. 14-33, 2004.

TEIXEIRA, J.C.; GUILHERMINO, R.L. Análise da associação entre saneamento e saúde nos estados brasileiros, empregando dados secundários do banco de dados indicadores e dados básicos para a saúde 2003 - IDB 2003. Eng Sanit Ambient., v. 11, n. 3, p.277-282, 2006.

Recebido em:17 de outubro de 2016 Aceito em:19 de dezembro de 2016 